\title{
Investigating interphase development in woodpolymer composites by inverse gas chromatography
}

\author{
TIMOTHY G. RIALS ${ }^{1}$ and JOHN SIMONSEN ${ }^{2}$ \\ 1 USDA Forest Service, 2500 Shreveport Highway, Southern Research Station, Pineville, \\ Louisiana 71360-5500, USA \\ 2 Departrnent of Forest Products, Oregon State University Corvallis, Oregon 97331, USA
}

Received 15 October 1999; accepted 15 December 1999

\begin{abstract}
The influence of secondary interactions on the development of interfacial structure in composites of wood and amorphous thermoplastic polymers is not well understood. This study used inverse gas chromatography to investigate the effect of different polymers on the surface energy of partially or fully coated white pine wood meal. In this way, the development of the interphase was monitored as a function of polymer depth on the wood surface. The polymers were selected to provide a range of functional groups and included polystyrene, poly(methyl methacrylate), poly(vinyl chloride), polymethacrylic acid, and polymethacrylonitrile. The overall variation of the dispersive component of the surface energy and the ratio of acceptor to donor coefficients appeared to group themselves into two categories based upon the polarity of the polymer's functional groups. In addition, the high loadings required for stabilization of the less polar polymers suggested that a relatively large volume of the matrix phase is affected by the wood filler.
\end{abstract}

Keywords: Inverse gas chromatography; surface energy; interface; wood composite.

\section{INTRODUCTION}

The combination of wood with a variety of synthetic polymer materials forms the foundation of a vast industrial base. From commodity materials like particleboard and plywood to engineered composites like laminated veneer lumber (LVL) and medium density fiberboard (MDF), this material combination offers an extremely versatile and affordable spectrum of products that are found in a variety of applications [I]. The focus on construction materials alone has restricted interest in the synthetic component to thermosetting polymers like phenol-formaldehyde, urea-formaldehyde, and isocyanate resins [2]. New opportunities undoubtedly exist when that perspective is expanded to consider thermoplastic polymers either as binders or matrix components. Composites of wood and thermoplastics also raise new questions regarding fundamental issues of adhesion, and the contribution of the interfacial component to performance properties. 
In contrast to the more conventionally utilized thermoset resins, commodity thermoplastics are high molecular weight, not reactive, and generally non-polar materials [3]. By virtue of these characteristics, the mechanisms for adhesion that are routinely relied upon in wood/thermoset composites, i.e. mechanical attachment and covalent bonding, are not available. As a result, inter-facial properties and ultimately composite performance are determined by specific interactions that are established across the boundary of the dissimilar phases [4]. The exact nature of these interactions and their impact on interphase structure and properties are poorly understood. Furthermore, the complexity of this system is highlighted by considering the multi-component nature of the wood substrate. Comprised of cellulose, hemicellulose, and lignin polymers, the chemical composition of the wood surface can vary dramatically from that of a carbohydrate to one dominated by aromaticity with functionality ranging from phenolic hydroxyl to carboxyl to carbonyl [5]. Additionally, extra-cellular components like fatty acids can effectively lend an olefinic character to the surface of the wood substrate [6,7]. At the very least, an extremely heterogeneous surface is to be expected.

This same surface complexity also promises considerable opportunity for engineering the surface to optimize interaction levels and manipulate fiber/matrix interfacial properties. This potential has been demonstrated to a limited degree for some systems, for example, the development of transcrystalline structure has been reported for wood fiber/polypropylene composites [8-10]. As a consequence of this behavior, the properties of this composite material are influenced by changes in matrix morphology that develop from direct interfacial interactions. In contrast, the effect of lignocellulosic fiber addition on the structure and properties of amorphous polymer matrices is less readily observed, and has been difficult to quantify experimentally. One potential approach was highlighted by studies on wood fiber surfaces grafted with methyl methacrylate [ 11 1] and maleic anhydride-modified polypropylene $[12,13]$. In these studies, inverse gas chromatography (IGC) was used to monitor the effect of polymer graft modification on fiber surface energetics. IGC might similarly offer a convenient and affordable technique to study interphase development in filled amorphous polymers where there are specific interactions, rather than covalent bonding.

Recently, Simonsen et al. [ 14] investigated the use of inverse gas chromatography to determine the variation in surface properties with polymer loading for idealized composites of wood particles and polystyrene. The work revealed some species dependence on surface properties of the composites, and suggested that the substrate influences a much greater volume fraction of polymer than would be expected if only a few monolayers of polymer comprised the interphase. The influences of the dispersive vs. polar nature and adhesive strength of secondary interactions present in the interphase between matrix polymer and discontinuous filler remains an important area that has been largely unexplored. This report, which builds on the earlier work, addresses this question by investigating the interfacial development 
of matrix polymers containing different functional groups and, therefore, different interaction mechanisms.

\section{EXPERIMENTAL}

White pine wood meal, mesh size \#20-60, was contributed by American Wood Fibers, Schofield, Wisconsin. The wood particles had an average $l / d$ ratio of 20 , and a surface area of $0.81 \mathrm{~m}^{2} / \mathrm{g}$. The polymers selected for study in the experiments included polystyrene (PS), poly(methyl methacrylate) (PMMA), poly(vinyl chloride) (PVC), poly(methacrylic acid) (PMAA) and polymethacrylonitrile (PMAN). They were obtained from Scientific Polymer Products and used as received. The composites were prepared by dissolving the appropriate amount of polymer in toluene and stirring for $24 \mathrm{~h}$. A portion (cu. 25\%) of the solution was then poured over a mound of the wood particles and allowed to dry under ambient conditions. The particles were then mixed, re-formed into a mound, and the process was repeated until the polymer solution was exhausted. The composites were dried under vacuum at $105^{\circ} \mathrm{C}$ and stored in a desiccator until used. Polymer composition was estimated by gravimetric measurements and FTIR spectroscopy.

The wood/polymer composite samples (cu. $20 \mathrm{~g}$ ) were packed into standard 6$\mathrm{mm}$ i.d., teflon, gas chromatography columns cut to a length of $1.2 \mathrm{tn}$. The columns were mounted in an integrator equipped Hewlett-Packard 5890 gas chromatograph with dual hydrogen flame ionization detectors maintained at $250^{\circ} \mathrm{C}$ to ensure flash vaporization of all probe vapors. Pre-purified nitrogen was used as the carrier gas at a flow rate of $20 \mathrm{ml} / \mathrm{min}$. Methane was used as the reference gas to determine column dead volume. Appropriate corrections for the pressure drop across the column were made on the basis of the pressure at the injection and outlet ports [ 16]. The columns were conditioned at $105^{\circ} \mathrm{C}$ for $24 \mathrm{~h}$ in the gas chromatograph prior to testing at $60^{\circ} \mathrm{C}$, which is well below the $T_{\mathrm{g}}$ of the polymers investigated, in order to minimize absorption of the probe in the polymer. The chromatography grade probe liquids used for IGC analysis were purchased from Aldrich Chemical Co., Milwaukee, WI, and were used as received. The probes and their physical properties are listed in Table 1. All retention times were the average of at least five injections for each column and condition investigated. All injections were less than $0.1 \mu \mathrm{l}$ to ensure a valid approximation of infinite dilution of the probe vapor.

\section{I. IGC data analysis}

The theory relating the retention time of a probe in a particular IGC column has been widely published $[6,15]$ and will only be summarized here. The equations of interest relate the retention volume, which is derived from the retention time as

$$
V_{\mathrm{N}}=j F\left(t_{\mathrm{r}}-t_{0}\right)
$$

where $V_{\mathrm{N}}=$ net retention volume; $j=$ a correction factor for gas compressibility [16]; $\mathbf{F}=$ the carrier gas flow rate; $t_{\mathrm{r}}=$ the retention time of the probe, and $t_{0}=$ 
the retention time of a marker probe (in our case, methane). $V_{N}$ is then related to the dispersive component of the surface free energy via

$$
R T \operatorname{In} V_{\mathrm{N}}=2 N_{\mathrm{A}}\left(\gamma_{\mathrm{s}}^{\mathrm{d}}\right)^{(1 / 2)} a\left(\gamma_{1}^{\mathrm{d}}\right)^{1 / 2}+\text { constant, }
$$

where $\mathbf{a}=$ the cross sectional area of the probe on the surface (a reported quantity); $\gamma_{1}^{\mathrm{d}}=$ the dispersive component of the surface tension of the probe (equal to the total surface tension for alkanes); $N_{\mathrm{A}}=$ Avogadro's number; $R=$ the gas constant; $T=$ the temperature $(\mathrm{K})$; and $\hat{\gamma}_{\mathrm{s}}=$ the dispersive component of the surface energy of the stationary phase of the IGC column for the case where all the probes are hydrocarbons. Thus, plots of $R T \ln V_{\mathrm{N}}$ vs. $a\left(\gamma_{1}^{\mathrm{d}}\right)^{1 / 2}$ for a series of alkanes yields a linear plot with a slope proportional to the dispersive component of the surface energy of the stationary phase of the IGC column.

Fowkes [ 17] proposed that the surface energy may be written as the sum of the components. Thus if we consider only dispersive and polar forces,

$$
\gamma^{\mathrm{T}}=\gamma^{\mathrm{d}}+\gamma^{\mathrm{p}}
$$

where $\mathrm{y}^{\mathbf{T}}=$ the total surface energy, $\gamma^{\mathrm{d}}=$ the dispersive component, and $\gamma^{\mathrm{p}}=$ the polar component of the surface energy. When probes other than alkanes are used, the retention volume is influenced by both components of the surface energy, and the values of $R T \ln V_{\mathrm{N}}$ 'for polar probes predictably fall above the line obtained for purely' dispersive probes. The difference between the ordinal value for the polar probe and the equivalent value for the alkane probe is defined as the specific interaction parameter, $\Delta G_{\mathrm{sp}}^{0}$, as follows

$$
\Delta G_{\mathrm{sp}}^{0}=R T \ln \frac{V_{\mathrm{N}}}{V_{\mathrm{N}}^{\text {ref }}},
$$

where $V_{N}^{\text {ref }}$ is the value of $V_{N}$ on the alkane reference line at the value of $\left[a\left(\gamma_{1}^{\mathrm{d}}\right)^{1 / 2}\right]$ corresponding to the specific probe of interest. The $\Delta G_{\mathrm{sp}}^{0}$ value for the basic

Table1.

Characteristics of inverse gas chromatography probes

\begin{tabular}{llllc}
\hline Probe & Area $\left(\AA^{2}\right)$ & $\gamma_{1}^{\mathrm{d}}\left(\mathrm{mJ} / \mathrm{m}^{2}\right)$ & $\begin{array}{l}\mathrm{AN} \\
(\mathrm{kcal} / \mathrm{mol})\end{array}$ & $\begin{array}{l}\mathrm{DN} \\
(\mathrm{kcal} / \mathrm{mol})\end{array}$ \\
\hline n-hexane & 51.4 & 18.4 & 0 & $\mathbf{0}$ \\
n-heptane & 57.0 & 20.3 & 0 & $\mathbf{0}$ \\
n-octane & 63.0 & 21.3 & $\mathbf{0}$ & $\mathbf{0}$ \\
n-nonane & 69.0 & 22.7 & $\mathbf{0}$ & $\mathbf{0}$ \\
n-decane & 75.0 & 23.4 & $\mathbf{0}$ & $\mathbf{0}$ \\
Specific & & & & $\mathbf{0}$ \\
Chloroform & 44.0 & 25.9 & 23.1 & 20.1 \\
Tetrahydrofuran & 45.0 & 22.5 & 8.0 & 12.5 \\
Acetone & 52.4 & 16.5 & 17.0 & \\
\hline
\end{tabular}


(tetrahydrofuran) probe was divided by the $\Delta G_{\mathrm{sp}}^{0}$ value for the acidic $\left(\mathrm{CHCl}_{3}\right)$ probe to obtain the basic : acidic ratio.

\section{RESULTS AND DISCUSSION}

The sensitivity of inverse gas chromatography to small differences in surface properties has been illustrated for a variety of materials [18], and may be used to resolve the individual components of the surface energy. Figure 1 illustrates the standard treatment of data for wood particles coated with 1, 5 and 15 wt\% PMMA. In all cases, a linear retention profile is found for the series of alkanes while retention times for the specific probes fall above this reference line. As the polymer loading is increased, the retention time of the probes is reduced slightly; however, there is very little change in the slope of the alkane reference. This behavior may reflect small changes in surface area of the stationary phase as the polymer restricts accessibility to interior surfaces of the wood substrate. The uncertainty associated with surface area determination due to the relatively low initial surface area of the particles, as well as the inherent variability of the BET process, made this question of changing surface area impossible to answer in this study.

At 1\% PMMA loading, the basic (tetrahydrofuran) probe had a greater retention volume when compared to either the acidic (chloroform) or amphoteric (acetone) probes. This suggests a slightly acidic surface since the basic probe is assumed to interact primarily with exposed acidic groups. As the polymer loading is increased to $5 \%$, the $V_{\mathrm{N}}$ of acetone is only slightly reduced and is comparable to the $V_{\mathrm{N}}$ values of both the acidic and basic probes. The reduction in $V_{\mathrm{N}}$ for each of the specific probes continues as the polymer content is raised to $15 \%$. This surface is best characterized as amphoteric, but the acidic and basic probes are now inverted, which indicates a clear shift in surface functionality. This is consistent with increased exposure of the methacrylate side chain as the polymer coating develops. It also
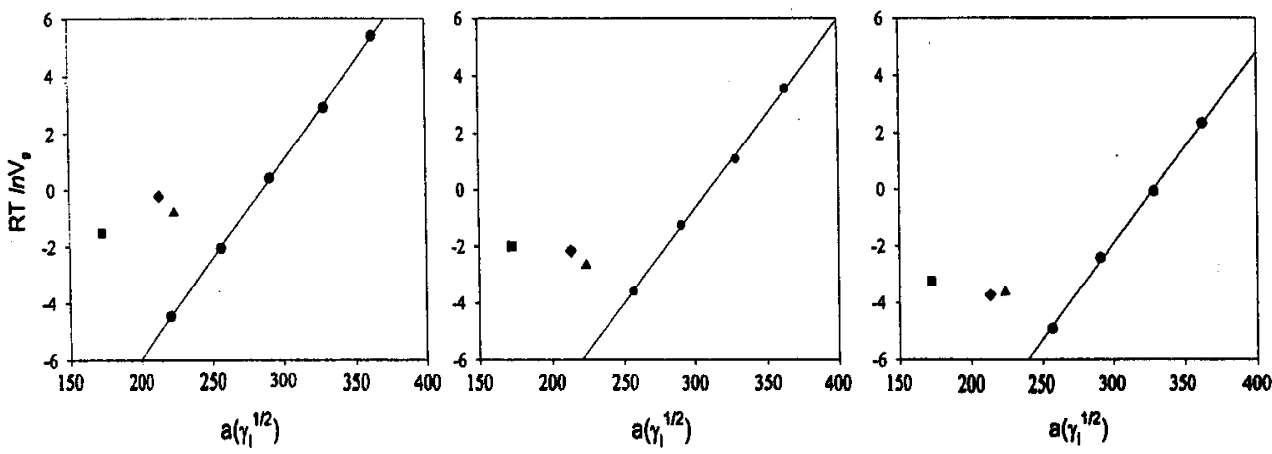

Figure 1. Plot of $R T \ln V_{\mathrm{g}}$ for determination of dispersive surface energy and specific interaction parameter for wood coated with 1 (left), 5 (middle) and 15 (right) percent poly(methyl methacrylate): alkanes

(•), chloroform (A), tetrahydrofuran

$(\boldsymbol{\nabla})$, and acetone $(\boldsymbol{D})$. 
illustrates the sensitivity of IGC to changes in surface chemical makeup of the developing composite.

\subsection{Dispersive interactions}

In accordance with equation (2), the dispersive component of the surface free energy $\left(\gamma_{\mathrm{s}}^{\mathrm{d}}\right)$ was calculated from the alkane probe data for the different polymer systems studied. The results are summarized in Table 2. The dispersive surface energy of the uncoated wood substrate (after exposure to the same conditions used in composite preparation) was determined as $32.8 \mathrm{~mJ} / \mathrm{m}^{2}$, in good agreement with the literature values $[11,19]$. Generally, the variation in this parameter with polymer content is relatively small for the polymer types investigated. Substantial variation in dispersive surface energy is observed only at the higher loading levels for polystyrene and poly(vinyl chloride), otherwise ranging between 30 and $34 \mathrm{~mJ} / \mathrm{m}^{2}$. This is not altogether surprising since the dispersive force contributions for the selected polymers (as judged from solubility parameter data) are not dramatically different from each other. The dispersive component of the three-dimensional solubility parameter ranges between lg.2 $(\mathrm{MPa})^{1 / 2}$ for PMAN and $21.28(\mathrm{MPa})^{1 / 2}$ for polystyrene [20]. Consequently, dramatic deviations in surface energy should not be expected as the polymer fraction of the surface is increased, and none were observed. Still, a closer look at the subtler variations is of interest.

The data relating $\gamma_{s}^{\mathrm{d}}$ to polymer loading in Table 2 are shown graphically in Fig. 2. It appears that at least two distinct behavioral patterns can be identified. First, the PMAN and PMAA are very similar in their profiles despite a more pronounced minimum at low loading of PMAA. Second, PVC and PMMA are also distinguished by their similar behavior. These two polymers are more cyclic in their behavior as a minimum is observed around 5\% and a second minimum appears imminent at higher polymer loadings. Polystyrene seems to be unique in its response, although some similarities exist to PVC and PMMA at lower polymer loadings. The continued rise in dispersive surface energy with polymer content is consistent with earlier observations for polystyrene on Douglas fir, which indicated a maximum value at $15 \%$ before declining significantly [14]. This characteristic profile may reflect absorption/penetration of the probes into the bulk polymer (a common source of error in IGC); however, no evidence of this was observed from the symmetry of the eluted peaks.

The significance of these comparisons rests upon the relative error of the observations. Each data point shown is the average of at least four probe injections, and the error bars shown are based on the standard deviations observed for each set of injections. They do not represent the deviation between IGC columns prepared separately. In separate experiments, identically prepared columns were run for selected systems and conditions (10\% PMMA and 5\% PS) and the variability in $\gamma_{\mathrm{s}}^{\mathrm{d}}$ was found to be less than $3 \%$. While this does not preclude the presence of systematic errors in the data shown, it does give a rough estimate of what might be expected in terms of error. Additionally, even if the absolute values of surface energy deter- 
Table 2.

Variation in the dispersive component of composite surface energy with polymer type and content

\begin{tabular}{llllll}
\hline $\begin{array}{l}\text { Polymer } \\
\text { (wt\%) }\end{array}$ & content & \multicolumn{5}{l}{ Dispersive surface energy, $\gamma_{\mathrm{s}}^{\mathrm{d}}\left(\mathrm{mJ} / \mathrm{m}^{2}\right)$} & \\
\cline { 2 - 6 } & PS & PMMA & PMAA & PMAN & PVC \\
\hline 0 & $\mathbf{3 2 . 8}$ & $\mathbf{3 2 . 8}$ & $\mathbf{3 2 . 8}$ & $\mathbf{3 2 . 8}$ & $\mathbf{3 2 . 8}$ \\
1 & $\mathbf{3 2 . 5}$ & $\mathbf{3 2 . 8}$ & $\mathbf{3 1 . 2}$ & $\mathbf{3 2 . 7}$ & $\mathbf{3 4 . 0}$ \\
3 & $\mathbf{3 2 . 2}$ & $\mathbf{3 1 . 4}$ & $\mathbf{3 2 . 8}$ & $\mathbf{3 2 . 6}$ & $\mathbf{3 3 . 4}$ \\
$\mathbf{5}$ & $\mathbf{3 4 . 9}$ & $\mathbf{3 0 . 3}$ & $\mathbf{3 3 . 1}$ & $\mathbf{3 2 . 3}$ & $\mathbf{3 3 . 2}$ \\
10 & $\mathbf{3 7 . 2}$ & $\mathbf{3 3 . 1}$ & $\mathbf{3 3 . 2}$ & $\mathbf{3 2 . 5}$ & $\mathbf{3 5 . 8}$ \\
15 & $\mathbf{4 0 . 1}$ & 31.3 & $\mathbf{3 4 . 4}$ & $\mathbf{3 3 . 7}$ & $\mathbf{3 5 . 2}$ \\
\hline
\end{tabular}

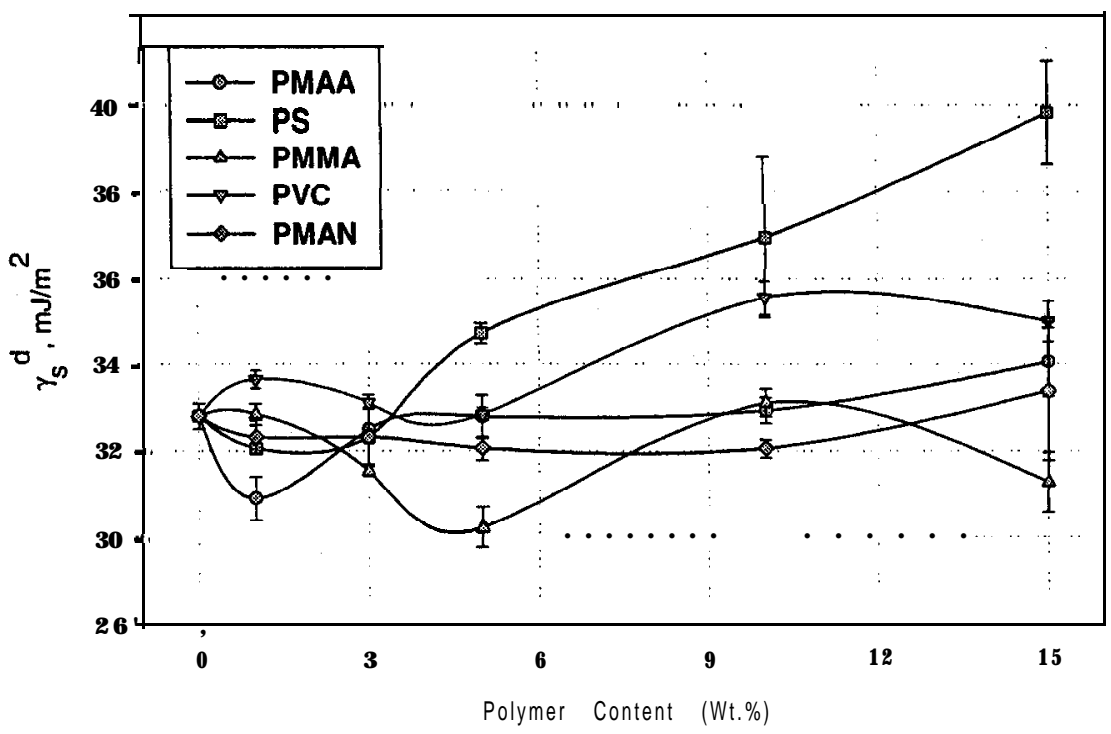

Figure 2. Variation in the dispersive surface energy $\left(\gamma_{\mathbf{s}}^{\mathrm{d}}\right)$ with polymer content for the different polymer systems: PMAA (0), PS (Cl), PMMA (A), PVC (V), and PMAN $(\diamond)$.

mined from these experiments contain systematic errors, relative comparisons based on the general trends observed should still be valid. With these limitations in mind, the observations suggest that the wood substrate influences the evolution of surface characteristics as polymer loading is increased, and the ultimate profile of $\gamma_{s}^{\mathrm{d}}$ vs. polymer loading varies with polymer functionality. The differences presumably arise as a consequence of specific interaction with the filler surface.

\subsection{Specific interactions}

The retention time of specific probes is determined by both dispersive and polar forces, which arise from Lewis acid/base interactions and hydrogen bonding between the probe molecule and column stationary phase. Because of this added polar contribution, the non-alkane probes are consistently displaced above the 
Table3.

Summary of specific interaction parameters $\left(\Delta G_{\mathrm{sp}}^{0}\right)$ for composites prepared with varying amounts of different polymer types

\begin{tabular}{|c|c|c|c|c|c|c|c|}
\hline \multirow[t]{2}{*}{ Polymer system } & \multirow[t]{2}{*}{ 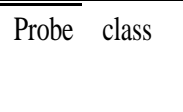 } & \multirow{2}{*}{$\frac{\text { Polymer }}{0}$} & \multicolumn{5}{|c|}{ composition (wt\%) } \\
\hline & & & 1 & 3 & 5 & 10 & 15 \\
\hline \multicolumn{8}{|l|}{ PS } \\
\hline & Amphoteric & 5.1 & 6.00 & 6.39 & 7.21 & 9.01 & 10.47 \\
\hline & Acidic & 3.5 & 3.94 & 4. 31 & 5.14 & 6.56 & 7.32 \\
\hline & Basic & 4.9 & 5.39 & 5.77 & 6.49 & 7.71 & 8.48 \\
\hline \multicolumn{8}{|l|}{ PMMS } \\
\hline & Amphoteric & 5.1 & 6.21 & 6.62 & 1.2 & 1. 34 & 7.32 \\
\hline & Acidic & 3.5 & 3.46 & 3.62 & 3.1 & 4.08 & 3.45 \\
\hline & Basic & 4.9 & 4.76 & 4.14 & 4. 32 & 4. 64 & 4.07 \\
\hline \multicolumn{8}{|l|}{ PMAA } \\
\hline & Amphoteric & 5.1 & 6.26 & 5.19 & 6.07 & 5.45 & 5.19 \\
\hline & Acidic & 3.5 & 2.95 & 2.90 & 3.22 & 3.28 & 3.31 \\
\hline & Basic & 4.9 & 4. 38 & 4.47 & 4.12 & 4.77 & 4.80 \\
\hline \multicolumn{8}{|l|}{ PMAN } \\
\hline & Amphoteric & 5.1 & 5.56 & 5.35 & 6.56 & 6.15 & 5.14 \\
\hline & Acidic & 3.5 & 3.54 & 3.36 & 3.50 & 3.36 & 3.25 \\
\hline & Basic & 4.9 & 5.11 & 4.94 & 4.97 & 4.80 & 4.64 \\
\hline \multicolumn{8}{|l|}{ PVC } \\
\hline & Amphoteric & 5.1 & 5.79 & 6.20 & 6.83 & 7.85 & 8.29 \\
\hline & Acidic & 3.5 & 3.23 & 3.39 & 3.04 & 4.28 & 4.15 \\
\hline & Basic & 4.9 & 4.88 & 5.1 & 4,71 & 6.20 & 6.06 \\
\hline
\end{tabular}

alkane reference line (Fig. 1), and the difference defines the specific interaction parameter, $\Delta G_{\mathrm{sp}}^{0}$. The results of this analysis are summarized in Table 3. The specific interaction parameters of the amphoteric, acidic and basic probes for the unmodified wood substrate were determined as 5.1, 3.5 and 4.9, respectively. As noted earlier, this suggests the wood surface is largely amphoteric with a somewhat acidic character (since the basic probe interacts with acidic sites). It is surprising that all three parameters show a consistent increase for the polystyrene composites, raising again the concern of bulk absorption in this system. For the more polar polymers (PMAA and PMAN), the acidic interaction parameter is more stable as the polymer content is increased, while both the amphoteric and basic components vary. The relatively low value of the acidic interaction parameter for PMAA and PMAN suggests a reduced availability of basic sites on the composite surface. However, more data are required before any definitive conclusions can be reached.

Figure 3 illustrates how polymer content influences the ratio of the $\Delta G_{\mathrm{sp}}^{0}$ values for the basic and acidic probes for PMAA, PMAN, and PS. Both the PMAA and PMAN composites exhibit a basic : acidic ratio maximum around 3 percent polymer loading before reaching a plateau around 6-7\% at a slightly higher value than the 


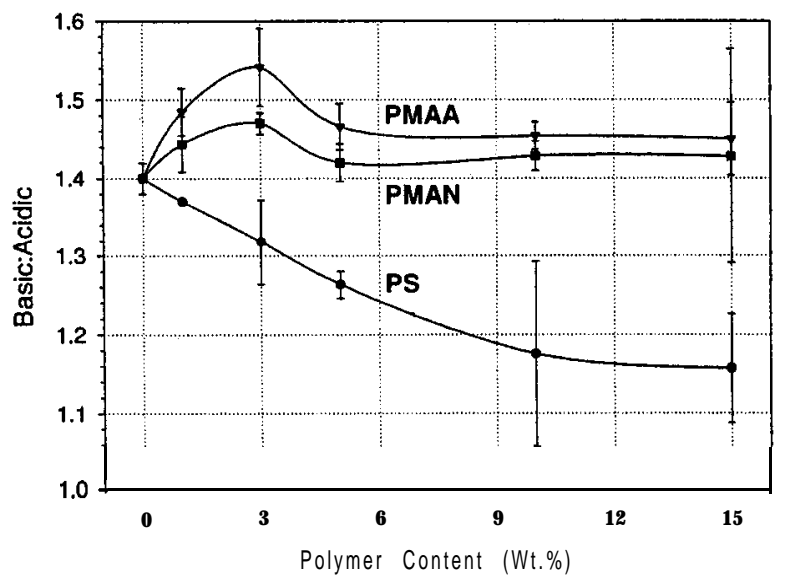

Figure 3. Effect of polymer addition on the relative contribution of the acidic and basic interaction

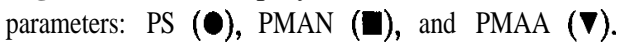

control wood sample. The PS data provide a sharp contrast to this behavior in that a substantial decrease in the ratio occurs before leveling off near 15\%. Also, PS does not exhibit a maximum at low polymer contents. As the coverage depth of PS increases, the basic : acidic ratio tends toward I, which might be expected for a largely non-polar surface. By contrast, the more polar polymers, PMAA and PMAN tend toward a more basic ratio, closer to that of the pure wood surface, and reflecting their intrinsically acidic character. It is difficult to explain the simila behavior of such different functionality polymers as PMAA and PMAN. It is also important to realize that we are looking at the adsorption phenomena of specific probes toward specific surfaces. Factors such as molecular configuration and the specific morphology of the polymer surface including orientation of the functional groups on it may play an important role in determining the adsorption, and the subsequent values for $\Delta G_{\mathrm{sp}}^{0}$. However, the dispersive nature of the less polar polymer, PS, is readily apparent.

Also apparent is the effect of polarity on the depth of the interphase. The more polar polymers, PMAA and PMAN, appear to be stabilized in basic : acidic ratio at about 5\% polymer loading. The PS appears to be reaching a plateau at about $15 \%$. Presumably, the stabilization of the acid : base ratio, and also the stabilization of the $\gamma_{\mathrm{s}}^{\mathrm{d}}$ values, which is roughly similar to the basic:acidic ratios, indicates the extent of the interphase in terms of average depth. While the limited data do not allow firm conclusions to be reached, it is clear that the different polymers show different interphase depths. Also, this depth may correlate to the interaction preference towards the specific probes, and depend at least partially upon the polarity of the polymer involved.

A plot similar to Fig. 3, but for the PVC and PMMA composites is shown in Fig. 4. The polystyrene data are included to provide a point of reference. As suggested by the variation in the dispersive surface energy, these two polymers are 


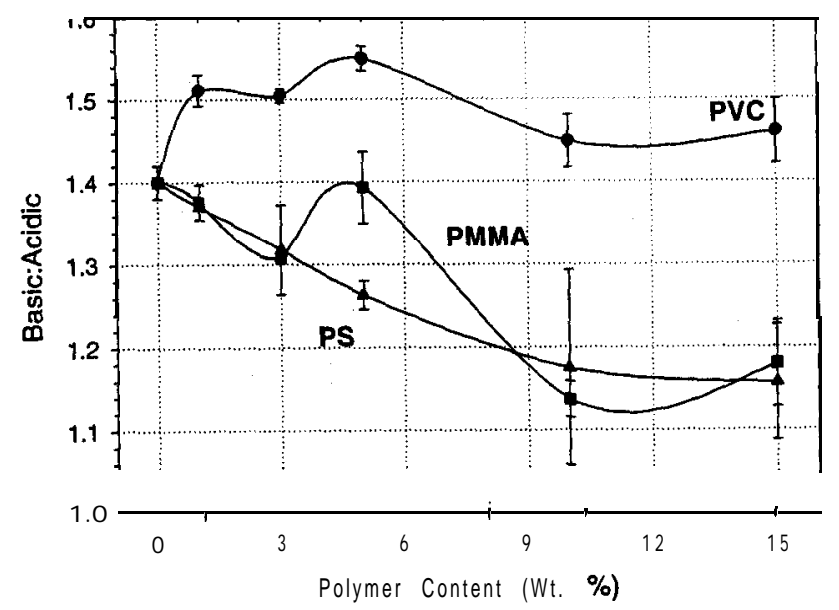

Figure 4. Effect of polymer addition on the relative contribution of the acidic and basic interaction

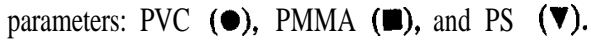

distinguished by a cyclic variation in the basic : acidic ratio as the polymer content is increased. In fact, the plot of $\gamma_{\mathrm{s}}^{\mathrm{d}}$ (Fig. 2) is roughly the mirror image of Fig. 4, i.e. when $\gamma_{\mathrm{s}}^{\mathrm{d}}$ is high, the basic : acidic ratio is low and vice versa. For PVC, the basic : acidic ratio increases significantly at low polymer levels. The behavior is consistent with the expectation that this polymer would preferentially interact with basic sites on the wood surface. The initial rise is followed by a second maximum at 5\% loading before decreasing at higher polymer levels. PMMA exhibits a similar response although the maximum at 5\% is much more pronounced. Also, there is a general decline in the basic : acidic ratio for PMMA which is similar to that for PS. As suggested in the discussion of Fig. 3, differences in interaction strength for the specific probes may partially account for these differing behaviors. While the limited number of data points do not allow definitive assignment of molecular significance to this observation, it does substantiate the idea that the wood substrate may influence structural organization in a large fraction of the polymer matrix.

The apparent oscillation of the PMMA curve about the PS curve leads to the speculation that dispersive forces are dominating in both systems. This is not what one might expect, based on PMMA's polarity and oxygen functionality, which is closer to PMAA, PMAN, and PVC than PS. At least with respect to adsorption of the probes used in this study, PMMA and PS behave similarly, although not identically. Prior results from dynamic mechanical analysis and differential scanning calorimetry [14] on PS support the contention that a large portion of the polystyrene matrix is influenced by the presence of wood and wood fiber. From the reduction in heat capacity and tan delta peak intensity, it was concluded that approximately 15 to $20 \%$ of the polymer fraction was removed from participating in the glass transition $\left(T_{\mathrm{g}}\right)$. These inverse gas chromatography data are consistent with that conclusion, and suggest that the presence of wood filler may enhance the 
level of structural order in some amorphous polymers. Additional studies are needed to answer this question for PMMA.

The maxima at low polymer for PMAA, PMAN, PVC, and PMMA suggest some sort of preferential adsorption is occurring. Furthermore, the oscillating curves for PVC and PMMA suggest an even more complicated adsorption mechanism. Unfortunately, the limited data available are inadequate to make firm conclusions. More study is needed to elucidate the specific adsorption mechanisms in these cases.

\section{CONCLUSIONS}

Inverse gas chromatography is a useful tool for qualitatively evaluating surface property differences during interphase development in wood/polymer composites. Despite relatively small changes in the dispersive surface energy, comparable profiles were detected for PMAN and PMAA. Both PMMA and PVC demonstrated similar behavior, as well. These two groups were also clearly resolved by basic : acidic ratios, using data generated from the retention of polar probes. The results indicate that the polar PMAA, PMAN, and PVC polymers interact specifically with contrasting sites on the wood substrate before reaching an equilibrium slightly above 5\% polymer loading. In contrast, the less polar polymers were more variable over the entire range of polymer loadings investigated. While the behavior suggests substantially different surfaces with polymer addition, the limited number of data points unfortunately restricts the significance of the observation. Even so, the results clearly indicate that the wood substrate influences the structure and the properties of more than the first several molecular layers of polymer, and point out the importance of chemical functionality in determining the properties of the interphase in wood-polymer composites.

\section{REFERENCES}

1. D. Fengel and G. Wegener, Wood: Chemistry, Ultrastructure, Reactions. W. de Gruyter, Berlin (1984).

2. A. Pizzi, Advanced Wood Adhesive Technology. Marcel-Dekker, Inc., New York (1994).

3. R. B. Seymour and C. E. Carraher, in: Polymer Chemistry, an Introduction, Third edition, p. 379. Marcel-Dekker, Inc., New York (1992).

4. Y. S. Lipatov and A. E. Nesterov, Macromolecules 8 (6), 889 (1975).

5. D. W. Gunnels, D. J. Gardner and M. P. Wolcott, Wood and Fiber Sci. 26 (4), 447 (1994).

6. F. P. Liu, T. G. Rials and J. Simonsen, Langmuir 14,536 (1998).

7. R. W. Hemingway, TAPPI 52 (11), 2149 (1969).

8. G. Han, S. Saka and N. Shiraishi, Mokuzai Gakkaishi 37 (3), 241 (1991).

9. D. T. Quillin, D. F. Caulfield and J. A. Koutsky, J. Appl. Polym. Sci. 50, 1187 (1993).

10. D. T. Quillin, M. Yin, J. A. Koutsky and D. F. Caulfield, J. Appl. Polym. Sci. 52,605 (1994).

11. D. P. Kamden and B. Riedl, J. Wood Chem. Technol. 11, 57 (1991).

12. A.C. Coupas, H. Gauthier and R. Gauthier, Polym. Composites 19,280 (1998).

13. L. M. Matuana, J. J. Balatinecz, C. B. Park and R. T. Woodhams, Wood and Fiber Sci. 31 (2), $116(1999)$. 
14. J. Simonsen, Z. Hong and T. G. Rials, Wood and Fiber Sci. 29, 75 (1997).

15. G. M. Dorris and D. G. Gray, J. Colloid Interf. Sci. 77,353 (1980).

16. A. E. Bolvari, T. C. Ward, P. C. Koning and D. P. Sheehy, in: Inverse Gas Chromatography, D. R. Lloyd, T. C. Ward, H. P. Schreiber and C. C. Pesana (Eds), p. 12. ACS Symposium series 391, American Chemical Society, Washington, DC (I 989).

17. F. M. Fowkes, J. Adhesion Sci. Technol. 1, 7 (1987).

18. J. M. Braun and J. E. Guillet, in: Advances in Polymer Science, Vol. 21, p. 107. Springer-Verlag, New York (1976).

19. Q. Shen, J. Nylund and J. B. Rosenholm, Holzforschung 52,521 (1998).

20. E. A. Grulke, in: Polymer Handbook, Third Edition, J. Brandrup and E. H. Immergut (Eds), p. VII, 556. John Wiley \& Sons, New York (1989). 